\title{
Neuroendocrine neoplasm
}

INSERM

\section{Source}

INSERM. (1999). Orphanet: an online rare disease and orphan drug data base.

Neuroendocrine neoplasm. ORPHA:877

Endocrine tumours, also referred to as neuroendocrine tumours (NETs), are defined by a common phenotype which is characterized by the expression of general markers (neuron specific enolase, chromogranin, synaptophysin) and hormone secretion products. These tumours may be localized in any part of the body and are generally discovered in nonspecific situations, i.e. not immediately suggestive of NETs (tests for inherited predisposition to tumours or for a clinical syndrome caused by abnormal hormone secretion). 